\title{
The prostatic utricle cyst with huge calculus and hypospadias: A case report and a review of the literature
}

\author{
Weigang Wang, MD; Yuantao Wang, MD; Dechun Zhu, MD; Pengfei Yan, MD; Biao Dong, MD; \\ Honglan Zhou, MD, PhD
}

Department of Urology, First Hospital of Jilin University, Jilin, China

Cite as: Can Urol Assoc J 2015;9(5-6):E345-8. http://dx.doi.org/10.5489/cuaj.2381 Published online May 13, 2015.

\section{Abstract}

Prostatic utricle cysts with calculus and hypospadias are rare. There are a few reported cases. We present a case of a prostatic utricle cyst with huge calculus in a 25 -year-old male. He had a history of left cryptorchidism and surgery for penoscrotal hypospadias in his infancy. He was referred for frequent micturition, urgency of urination, urine pain, terminal hematuria, and dysuria. A computed tomography (CT) revealed a retrovesical cystic lesion of low density, showing a $5 \times 5$-cm calcification. Retrograde urethrocystography showed a $5 \times 5-\mathrm{cm}$ high-density shadow in the posterior urethra. The cyst was incised by transperineal approach and the stone was clearly observed and removed. Urethral stricture repair was performed simultaneously. The patient recovered smoothly after surgery.

\section{Introduction}

Prostatic utricle cysts are embryological remnants of the Müllerian duct, with prevalence rates as high as $4 \%$ and $1 \%$ in newborns and adults, respectively. ${ }^{1}$ There are few reported cases. $^{2-5}$ The cysts may be accompanied by other deformities, including hypospadias, renal agenesis, and incomplete testicular descent. The anatomical relationship of the prostatic utricle with adjacent structures, including bladder, rectal, ejaculatory duct and neurovascular structures, is relatively complicated, which may lead to a difficult treatment. This report describes an adult man with huge calculus secondary to prostatic utricle cyst diagnosed by computed tomography (CT) and retrograde urethrocystography.

\section{Case report}

A 25-year-old male presenfted with frequent micturition, urgency of urination, urine pain, terminal hematuria, and dysuria for 2 years. He had a history of left cryptorchidism and surgery for penoscrotal hypospadias in his infancy. A digital rectal examination showed a rocky, well-defined prostate with disappeared central sulcus. Abdominal CT demonstrated a retrovesical cystic lesion of low density, in which a $5 \times 5$-cm calcification was shown. The prostate and bilateral seminal vesicle glands were obscure (Fig. 1). Retrograde urethrocystography showed a $5 \times 5-\mathrm{cm}$ highdensity shadow in the posterior urethra. The urethra displayed a rough intima with beaded changes after the injection of contrast media. The contrast agent failed to flow into the bladder (Fig. 2). Urine culture showed Escherichia coil infection. Treatment with levofloxacin, for this infection, began 2 days before surgery. Blood test results and prostate-specific antigen were normal, and ejaculation function was normal. Semen analysis revealed a spermiogram with a volume of $3.5 \mathrm{~mL}$, a number of $42 \times 10^{6} / \mathrm{mL}$, motility of $75 \%$, teratospermia of $9 \%$, and $1 \times 10^{6} / \mathrm{mL}$ leukocytes. There were no erythrocytes on microscopic evaluation. Base on the examinations, the patient was diagnosed the prostatic utricle cyst. Urine cultures were negative on postoperative day 3. Since hypospadias was present, we performed a transperineal cyst incision.

The patient was placed in the lithotomy position under general anesthesia. First we explored the urethra with the 12th urethral bougie; the urethral stricture was about $10 \mathrm{~cm}$ from the urinary meatus and the bougie could not pass through the stricture (Fig. 3). The cyst was approached through a longitudinal incision of about $8 \mathrm{~cm}$ at the perineum (Fig. 4). While it was cut, yellow purulent secretions flowed out of the cyst and a spherical brown stone, about $5 \mathrm{~cm}$ in diameter, appeared and was removed completely (Fig. 5). Urethral stricture repair was performed after the fortified iodine solution washed the cyst. The patient had an uneventful recovery and the retrograde urethrocystography showed no evidence of urethral narrowing and stones 3 months postoperatively (Fig. 6). 

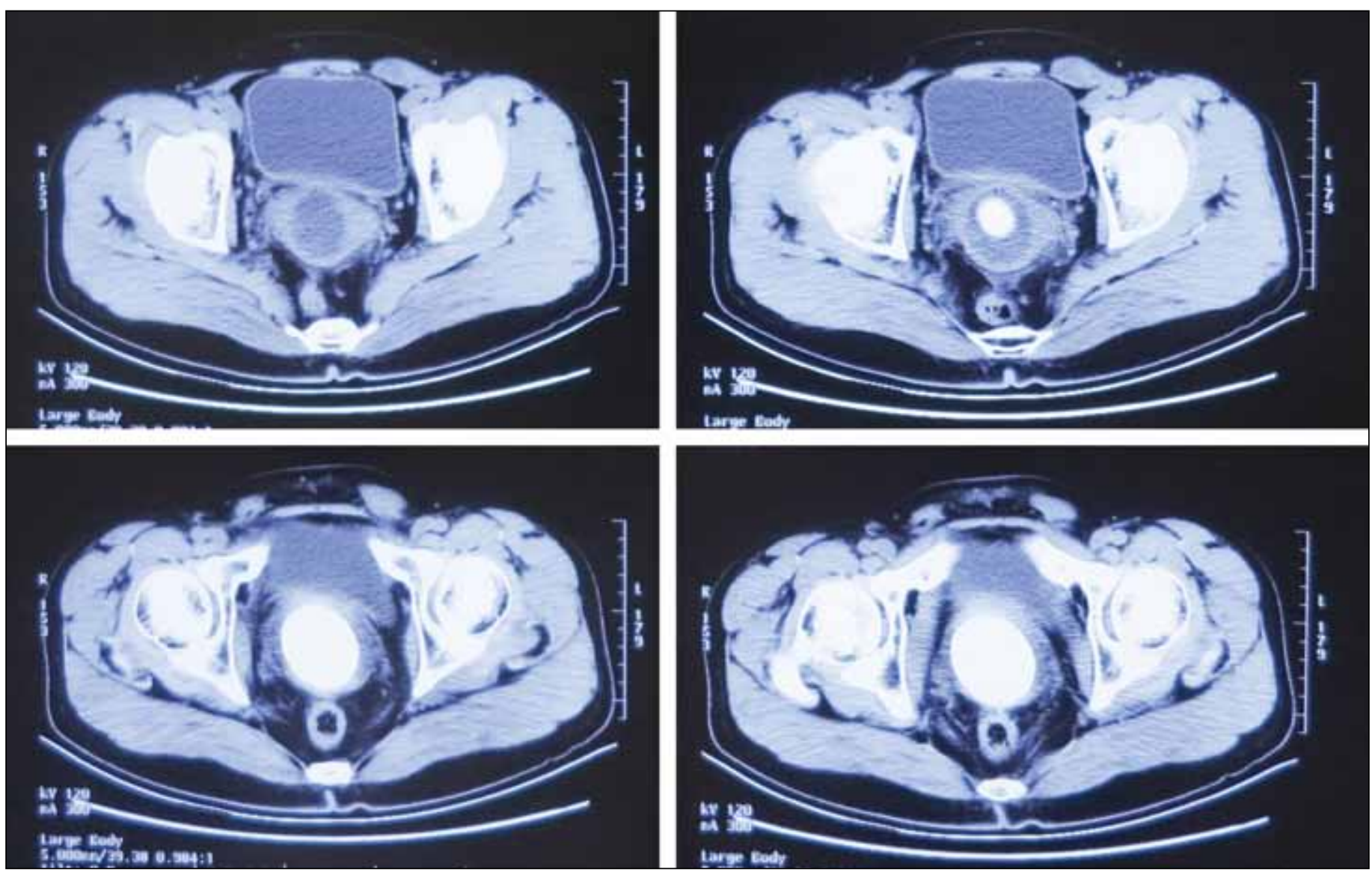

Fig. 1. A computed tomography scan showing a retrovesical cystic lesion of low density, in which a $5 \times 5$-cm calcification is seen. The prostate and bilateral seminal vesicle glands were obscure.

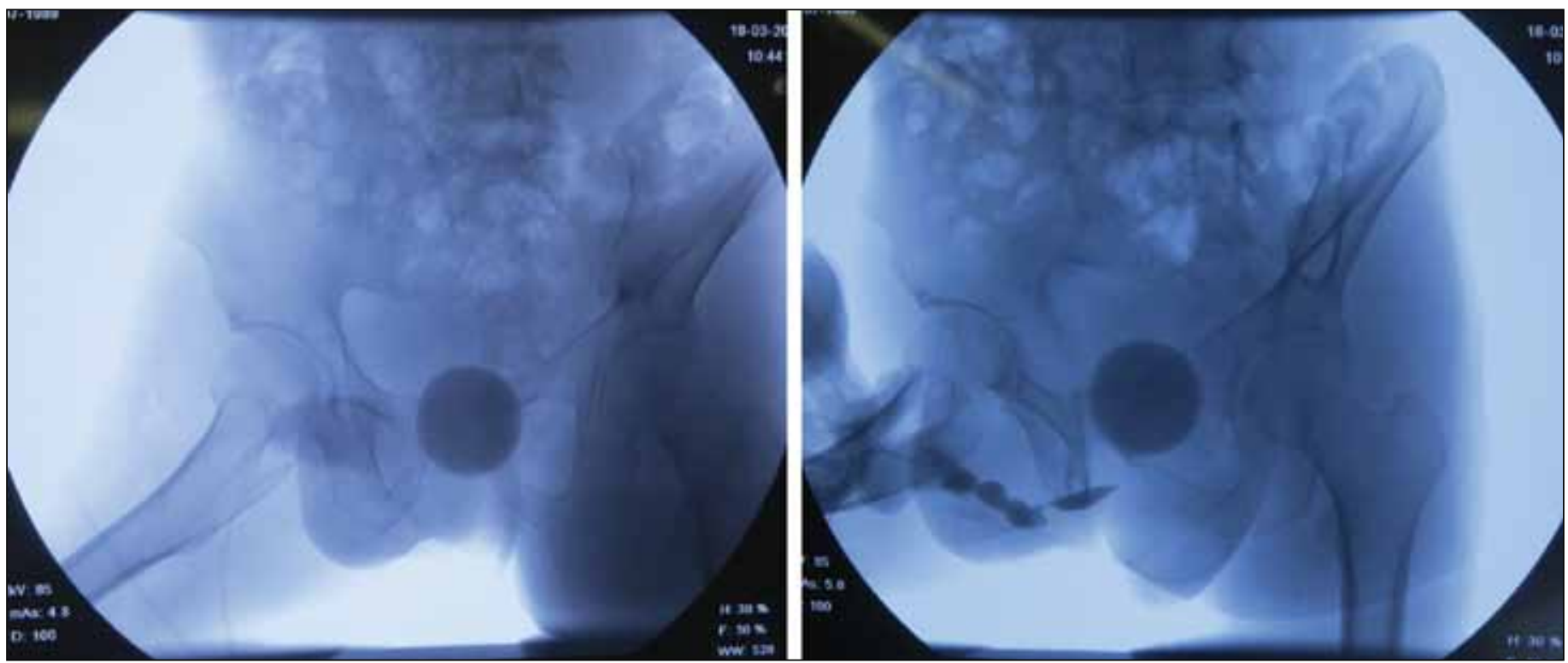

Fig. 2. Retrograde urethrocystography showing a $5 \times 5-\mathrm{cm}$ high-density shadow in the posterior urethra. The urethra displayed a rough intima with beaded changes after injection of contrast media. The contrast agent failed to flow into the bladder. 


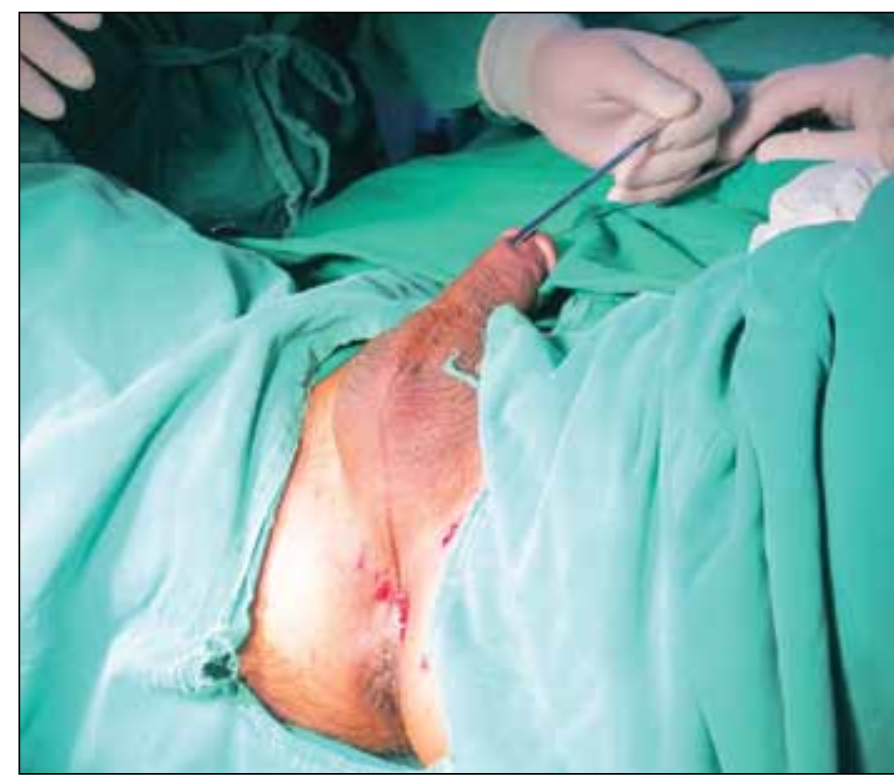

Fig. 3. The urethra was explored using the 12th urethral bougie and the urethral stricture was about $10 \mathrm{~cm}$ from the urinary meatus.

\section{Discussion}

Prostatic utricle cysts result from the regression of the Müllerian paramesonephric ducts or decreased androgenic stimulation of the urogenital sinus. The cyst can be congenital and often associated with hypospadias, cryptorchidism, or renal agenesis. The incidence of prostatic utricle cysts is about $11 \%$ to $14 \%$ in patients with hypospadias or sexual deformity, while $50 \%$ of perineal hypospadias comes with prostatic utricle cysts. ${ }^{6}$

The cyst is usually located in the midline, posterior to the prostate. It can become large and displace the urinary bladder or rectum, leading to various symptoms, including repeated urinary tract infection, epididymitis, hematuresis, pyuria, uroclepsia, oligospermia, uroschesis, and constipation. ${ }^{1,7}$ In this case, frequent micturition, urgency of urination, urine pain, terminal hematuria, and dysuria were present, but semen analysis was normal.

Since the cysts were close to the pelvic nerves, ureters, rectum, ejaculatory ducts and vas deferens, surgery was a challenge. Endoscopic transurethral puncture aspiration to the cysts or cyst unroofing had been reported by Schuhrke, but had a high recurrence rate. ${ }^{8}$ Open surgery has been used for most symptomatic utricle cysts, including transurethral unroofing and holmium laser litholapaxy, ${ }^{2,3}$ or complete resection through a suprapubic extraperitoneal approach. ${ }^{4,5}$ These approaches are accompanied by limited anatomical visualization, leading to increased difficulty in operation. In the present case, the cyst was accompanied by hypospadias and a huge stone, and the transurethral fenestration could

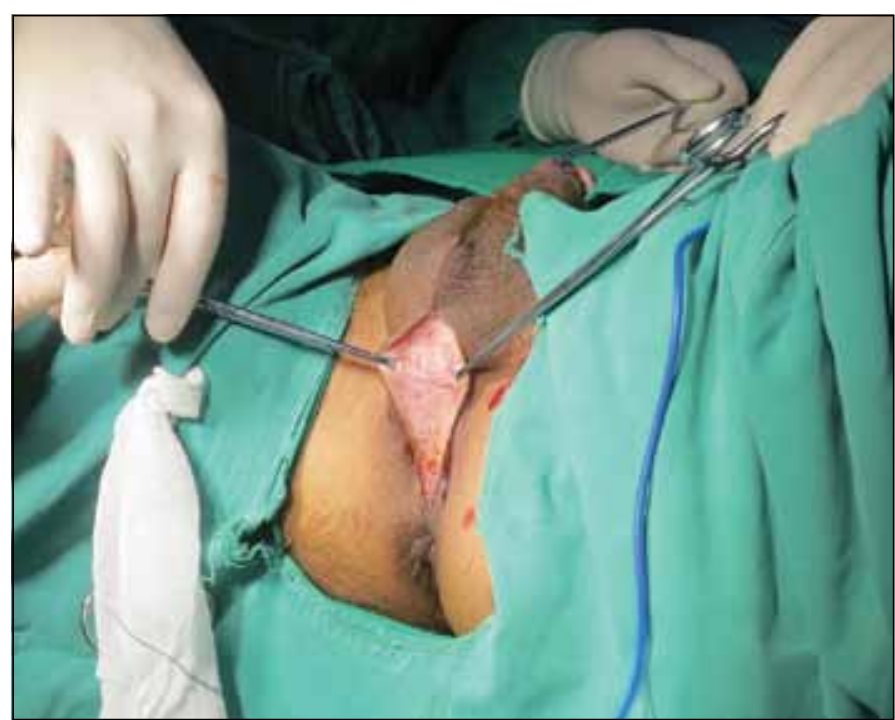

Fig. 4. A midline sagittal incision was made from the caudal end of the scrotum to the anterior margin of the anus.

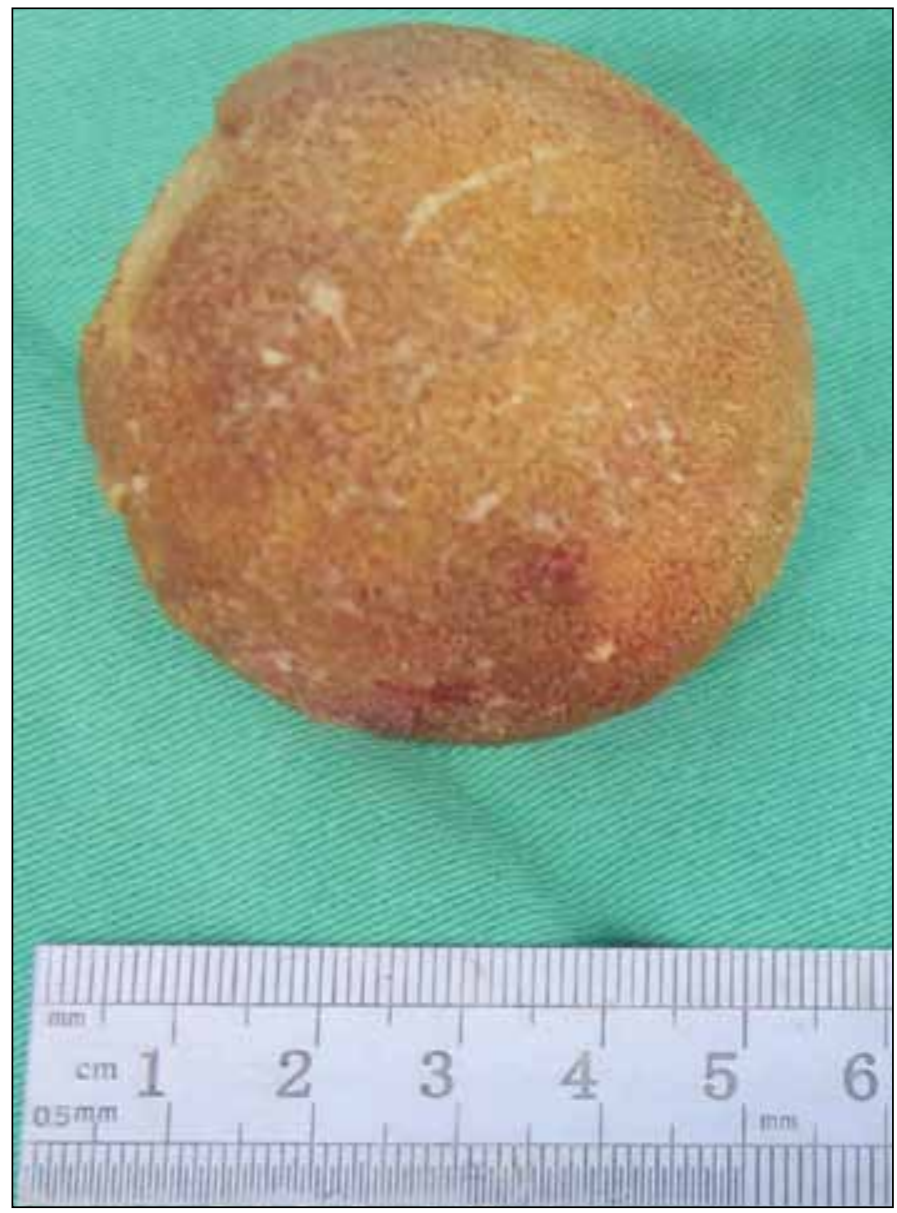

Fig. 5. The stone inside the cyst was about $5 \mathrm{~cm}$ in diameter. 
Wang et al.

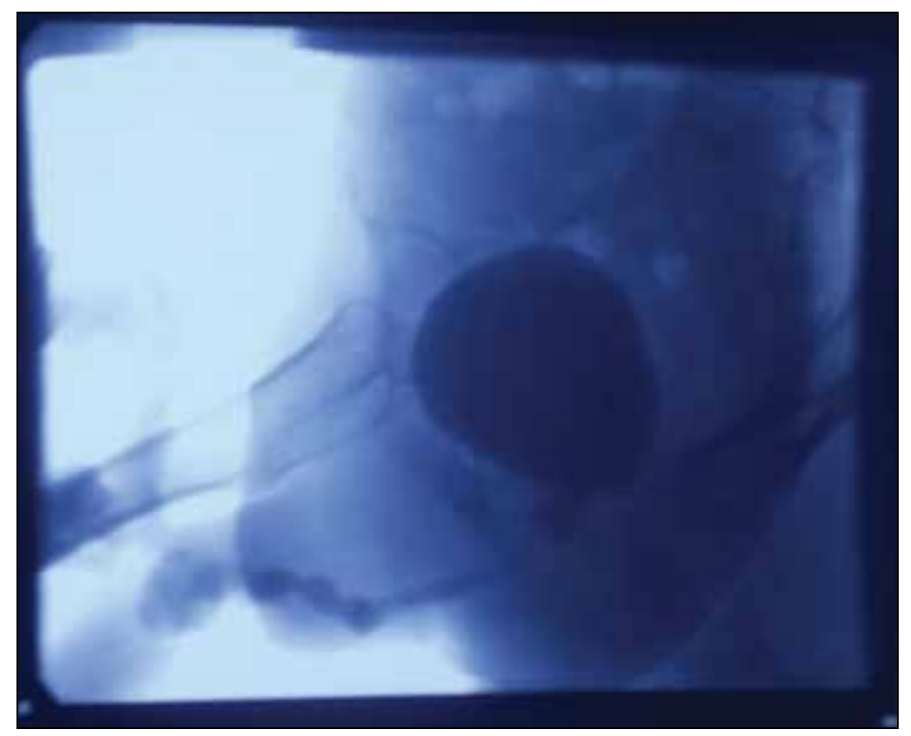

Fig. 6. Retrograde urethrocystography showing no evidence of urethral narrowing and stones 3 months after surgery.

not be completed because of the urethral stricture. Therefore the transperineal approach was adopted.

\section{Conclusion}

The prostatic utricle cyst is a rare clinical entity, and optimal surgical results require accurate definition of its anatomic relationship to the adjacent structures.
Competing interests: The authors declare no competing financial or personal interests.

This paper has been peer-reviewed.

\section{References}

1. Desautel MG, Stock J, Hanna MK. Müllerian duct remnants: Surgical management and fertility issues. J Urol 1999;162 (3 Pt 2):1008-13; discussion 1014. http://dx.doi.org/10.1097/00005392-19990900000012

2. Salvarcı A, Istanbulluoğlu 0 . Monosymptomatic persistent hematospermia due to rarely encountered prostatic utricle stones. Urol Int 2014 Jan 11. http://dx.doi.org/10.1159/000354766

3. Bagrodia A, Gerecci D, Ramirez D, et al. Holmium laser endourethrotomy and litholapaxy of an occult prostatic utricle calculus. Can J Urol 2012;19:6477-9.

4. Valdevenito JP, Valdevenito R, Cuevas M, et al. Cyst of the prostatic utricle: Report of a case complicated by giant lithiasis [in Spanish]. Arch Esp Urol 2002;55:960-2.

5. Spence HM, Chenoweth VC. Cysts of prostatic utricle (müllerian duct cysts): Report of two cases in children, each containing calculi, cured by retropubic operation. J Urol 1958;79:308-14.

6. Coppens L, Bonnet $P$, Andrianne $R$, et al. Adult Müllerian duct or utricle cyst: Clinical significance and therapeutic management of 65 cases. J Urol 2002;167:1740-4. http://dx.doi.org/10.1016/500225347(05)65190-7

7. Ramachandra M, Bendre PS, Redkar RG, et al. Isolated prostatic utricle. I Indian Assoc Paediatr Surg 2009;14:228-9. hitp://dx.doi.org/10.4103/0971-9261.59610

8. Schuhrke TD, Kaplan GW. Prostatic utricle cysts (Müllerian duct cysts). J Urol 1978;119:765-7.

Correspondence: Dr. Honglan Zhou, Department of Urology, First Hospital of Jilin University, №.71 Xinmin Street, Changchun 130021, Jilin, China; zhouhonglanjdyy@163.com 